\title{
Tolerability Study
}

National Cancer Institute

\section{Source}

National Cancer Institute. Tolerability Study. NCI Thesaurus. Code C98791.

A type of safety study that assesses the deg ree to which overt adverse effects can be tolerated by the subject. 